\title{
The Effects of Reading and Listening to the Heavenly Books on Heart Rate Variability
}

\author{
M aysam M ashhadimal ek ${ }^{1}$, N ader J afarnia D abanloo ${ }^{1}$, Shahriar Gharibzadeh ${ }^{2,3}$, Saman Parvaneh ${ }^{4}$ \\ ${ }^{1}$ Department of Biomedical Engineering, Science and Research B ranch, Islamic A zad U niversity, \\ Tehran, Iran \\ ${ }^{2}$ Institude for cognitive and brain sciences, Shahid B eheshti U niversity, Tehran, Iran \\ ${ }^{3}$ B asir Eye Research Center, Tehran, Iran \\ ${ }^{4}$ Philips Research N orth A merica, Cambridge, M A, USA
}

\begin{abstract}
The aim of this research is to test the effects of reading and listening to heavenly religious books on HRV (heart rate variability). To do this research, the ECG signal was recorded from the volunteers during four stages, and HRV signal were obtained. Then 12 features, including time domain, frequency domain, and nonlinear analysis, were extracted for each volunteer. By calculating P-value for each interference stage compared to the resting stage, we extracted four various features from HRV that changed during the interference. The P-values of these features were below 0.065 .

These 4 significant features of HRV were as follows: 1) $L F$ : low frequency of HRV signal. 2) RMSSD: the rootmean square difference of successive $R-R$ intervals. 3) SDNN: a standard deviation of the NN intervals, which is the square root of their variance. 4) SD1: one of the indices obtained from Poincaré plot.

According to the result, RMSSD, SDNN, and SD1 were significantly decreased and LF was significantly increased during the test. The results showed that the ANS (autonomic nervous system) activity in volunteers was changed during the test. This shows the mental or emotional activity when the volunteers are reading and listening to the holy books.
\end{abstract}

\section{Introduction}

\subsection{Heart Rate Variability}

The part of the nervous system that controls the most visceral functions of the body is the autonomic nervous system (ANS). The ANS can change visceral functions' rapidity and intensity. The two main subdivisions of the ANS are the sympathetic and parasympathetic nervous systems. Sympathetic/parasympathetic stimulation causes excitatory effects in some organs, but inhibitory effects in others [1].

There is a kind of oscillation in cardiac rhythm under the influence of the sympathetic and parasympathetic nervous systems, which reflect the autonomic nervous system's function [2]. The differences between each of the two consecutive heart pulses are called the heart rate variability [3]. The analysis of HRV can be used as a monitoring tool for autonomic nervous system changes [4]. As shown in Figure 1, HRV is a time series that is derived from the $R R$ interval in ECG.

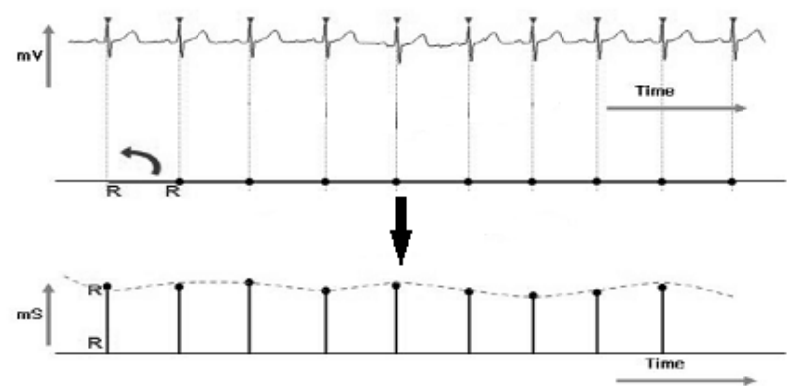

Figure 1. HRV signal are obtained using $R R$ values from the ECG signal.

\subsection{ANS stimulation by reading and listening to the Quran}

The Quran is a rhythmic prose text. It's melody comes from a combination of words and letters that are based on precise fine and sacred meanings. When the Quran is recited, many feel that the words and rhythm profoundly influence their mental and emotional state. It is widely regarded as the finest and most distinguished text in classical A rabic literature. Many studies have been undertaken by psychologists and hospital practitioners regarding the effects of the Quran and report that hearing the Q uran reduces the pain and anxiety of patients [5]. 
It has been observed that stimuli such as reading a religious book or listening to music can result in a significant change in the HRV signal. For example, $K$ urita et al. have evaluated the effects of reading religious books on elderly persons for a month [6]. They concluded that activities that stimulate spiritual reflection could help the health of the elderly [6].

Furthermore, the effects of various types of music on the HRV signal have been observed. For example, A maral et al. have studied the effects of auditory stimulation by different music genres on HRV [7]. Moreover, Iwanaga et al. have studied HRV with exposure to music repetition [8]; and Chuang et al. have examined the effects of music therapy on mental health and HRV in cancer patients [9].

We wish to build upon these previous studies by observing the combined effects of reading and listening to the Quran.

\section{2. $\quad$ Method}

In this experiment, 31 healthy subjects of the native Farsi-speaking M uslim people with a low understanding of the Arabic language, including 15 males and 16 females with no history of cardiovascular disease, aged 25 to 40 years, volunteered.

\subsection{Signal acquisition}

The ECG Signal was recorded from volunteers using the electrocardiography. The volunteer was placed in a quiet environment on a comfortable chair. A monitor was used for displaying texts and images. An adjustable volume head set was used to broadcast the recitation of the Quran. To increase the impact, specific verses with meanings of divine reward and resurrection were selected from the Quran. A s the Q uran was recited, the text of the verses was displayed on the monitor with Persian subtitles. At all stages, the volunteers were asked to pay attention to the meaning of the Q uran verses.

The steps for recording the ECG signal from volunteers are shown in Figure 2. The duration of each step was 5 minutes. Between each stage, breaks were taken in order to mitigate any crossover effect from the previous stage of the study. According to Figure 2, the acquisition protocol included four stages for ECG signal recording as follows: stage 1: while the volunteer is resting; stage 2: while the volunteer is reading the Quran, without listening to a recording; stage 3 : while the volunteer is listening to the Quran, without reading it (in this stage they look at a blank monitor); stage 4: while the volunteer is both reading and listening to the Quran.

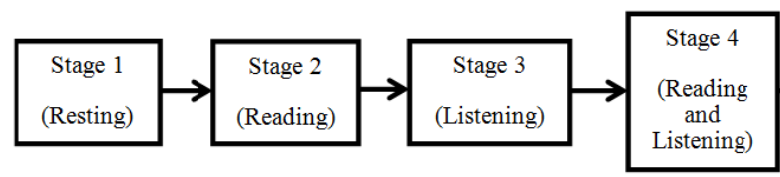

Figure 2. The Stages of ECG signal recording.

\subsection{Feature extraction}

After the ECG signal recording, RR signals were calculated. Then 12 features, including time domain, frequency domain, nonlinear analysis, and geometric methods, were extracted with MATLAB, as well as the Kubis program, for each volunteer.

Subsequently, using SPSS software, we calculated an independent t-test between each test stage and resting stage and for all HRV features, to determine the statistical significance of the mean differences at each stage compared to resting stage for volunteers.

\section{Results}

According to the t-tests performed at different stages of our experiments and the comparison of the $p$-values in each interference stage compared to the resting stage, 4 features were selected which were significantly increased or decreased during the test. As is shown in Table 1, all of the significant features have $p<0.065$ between fourth stage and the first stage (resting). A Iso, Table 2 shows the average of significant features in each stage.

These 4 significant features of HRV were as follows: 1) LF: low frequency of HRV signal. 2) RMSSD: the root-mean square difference of successive $R-R$ intervals. 3) SD N N : a standard deviation of the N N intervals, which is the square root of their variance. 4) SD1: one of the indices obtained from Poincaré plot. It is the standard deviation of the instantaneous beat-to-beat RR interval variability.

Table 1. P-value between each stage of the test and resting stage for significant features.

\begin{tabular}{llll}
\hline & Stage $1-2$ & Stage $1-3$ & Stage1-4 \\
\hline LF & 0.1 & 0.572 & $0.065^{* *}$ \\
RMSSD & 0.343 & 0.764 & $0.052^{* *}$ \\
SDNN & $0.040^{*}$ & 0.178 & $0.014^{*}$ \\
SD1 & 0.340 & 0.762 & $0.052^{* *}$ \\
& & & \\
& & & \\
${ }^{*}$ Statistically significant $(\mathrm{P}<0.05)$ & &
\end{tabular}

Table 2. The average of the four significant features in each stage.

\begin{tabular}{lllll}
\hline & Stage 1 & Stage 2 & Stage3 & Stage4 \\
\hline LF & 34.60 & 38.93 & 36.09 & 39.38 \\
RMSSD & 41.54 & 38.32 & 40.54 & 34.07 \\
SDNN & 49.84 & 44.70 & 46.85 & 42.18 \\
SD1 & 29.42 & 27.13 & 28.71 & 24.122 \\
& & & & \\
\hline
\end{tabular}




\section{Discussion}

In this study, we recorded the ECG signal from 31 M uslim volunteers in four stages. First, was the resting stage. Second, volunteers read the Quran, without listening to it. Third, volunteers listened to the Quran without reading it. Fourth, volunteers both listened to and read the Quran simultaneously. A fter obtaining the HRV signal, 12 features for each volunteer was extracted in four stages of the experiment. Finally, according to the statistical tests between the each stage and the resting stage, four features were obtained (see Table 1). These features that significantly differed between the some stage and the resting stage were:

1) LF: This feature was significantly different during the fourth stage in compared to resting stage. As indicated, this value was significantly increased at the fourth stage (see Table 2). As we know, increasing the LF indicates the decreased parasympathetic nerve function.

2) RMSSD: This feature was significantly different during the fourth stage in compared to resting stage. As indicated, this value was significantly decreased at the fourth stage (see Table 2).

3) SDNN: feature was significantly different during the following stages in compared to resting stage: the second stage and the fourth stage. A s indicated, this value was significantly decreased at the fourth stage (see Table 2).

RMSSD and SDNN associated with rapid short-term changes in heart rate, and show the parasympathetic effect of vagus nerve on HRV [11].

4) SD1: This feature was significantly different during the fourth stage in compared to resting stage. As indicated, this value was significantly decreased at the fourth stage (see Table 2). SD1 is a measure of rapid changes in $\mathrm{R}-\mathrm{R}$ intervals and shows the parasympathetic effect on sino-atrial node [10].

According to RMSSD, SDNN, and SD1 decreasing and LF Increasing, parasympathetic nerve stimulation decreases during the fourth stage of the test. This may be due to either increased mental or emotional activity when the volunteers were reading and listening to the Quran in the experiment.

\section{References}

[1] A.C. Guyton and J.E. Hall, "Textbook of Medical Physiology," Philadelphia, Penn: Elsevier/Saunders, 2006.

[2] M. Malik and A.J. Camm, "Heart rate variability," Cardiovasc Res, 61, 448-460, 2004.

[3] E.H. Hon and Lee, S.T, "Electronic evaluations of the fetal heart rate patterns preceding fetal death. Further observations," Am J Obstet Gynec, 87,814-26, 1965.

[4] M. Iwanaga, A. Kobayashi, and C. Kawasaki, "Heart rate variability with repetitive exposure to music," Biological
Psychology, 70, 61-66, 2005.

[5] M. Vaghefi, A. Motie Nasrabadi, S.M. Hashemi Golpayegani, M.R. Mohammadi, and S. Gharibzadeh, "Spirituality and brain waves," Journal of Medical Engineering \& Technology, 39:2, 153-158, 2015.

[6] A., K urita, B. Takase, , N. Shinagawa, E. Kodani, K. Okada, S. Iwahara, Y. Kusama, and H. A tarashi, "Spiritual activation very elderly individuals assessed as heart rate variability and plasma IL/10/IL-6 ratios," Int heart $j, 52$, 299-303, 2011.

[7] J. A maral, H. Guida, L. A breu, V. Barnab, F. Vanderlei, and V. Valenti, "Effects of auditory stimulation with music of different intensities on heart period," Journal of Traditional and Complementary Medicine, 6, 23-28, 2016.

[8] D.J. Ewing, C.N. M artin, J.R. Y oung, and B.F. Clarke, "The value of cardiovascular autonomic function tests: 10 year experience in diabetes," Diabetic care, 8, 491-8, 1985.

[9] C. Chuang, W. Han, P. Li, and S. Y oung, "Effects of music therapy on subjective sensations and heart rate variability in treated cancer survivors: A pilot study," Complementary Therapies in Medicine, 18, 224-226, 2010.

[10] R.A. Hoshi, C.M . Pastre, L.C. V anderlei, and M .F. Godoy, "Poincaré plot indexes of heart rate variability: relationships with other nonlinear variables," Autonomic Neuroscience: Basic and Clinical, 177, 271-274. 2013.

[11] C.M. DeGiorgio, P. M iller, S. M eymandi, A . Chin, J. Epps, S. Gordon, J. Gornbein, and RM. Harper, "RM SSD, a M ea sure of Heart Rate Variability, Is Associated With Risk Factors For SUDEP: The SUDEP-7 Inventory," Epilepsy Behav, 19(1), 78-81, 2011.

A ddress for correspondence:

Nader J afarnia Dabanloo

Department of Biomedical Engineering, Science and Research Branch, Islamic Azad University, Daneshgah Blvd, Simon Bulivar Blvd, Tehran, Iran

jafarnia@srbiau.ac.ir 